\title{
The clinical significance of the Ezrin gene and circulating tumor cells in osteosarcoma
}

This article was published in the following Dove Press journal:

OncoTargets and Therapy

8 February 2017

Number of times this article has been viewed

\author{
Guang-Xian Zhong' \\ Shao-Dan Feng ${ }^{2}$ \\ Rongkai Shen' \\ Zhao-Yang Wu' \\ Fei Chen' \\ Xia Zhu' \\ 'Department of Orthopaedics, \\ ${ }^{2}$ Department of Emergency, The First \\ Affiliated Hospital of Fujian Medical \\ University, Fuzhou, People's Republic \\ of China
}

Purpose: The aim of this study was to investigate the clinical significance of circulating tumor cells (CTCs) in the peripheral blood of an osteosarcoma and the Ezrin gene expressed in CTCs. Patients and methods: CTC enrichment was done with $\mathrm{CanPatrol}^{\mathrm{TM}} \mathrm{CTC}$ enrichment technique in 41 patients with osteosarcoma. The characterization of CTCs was performed using a multiple messenger RNA in situ analysis (MRIA). The expression of the Ezrin gene in CTCs was detected by RNA probe technology. The correlations of CTC counts, cell type and the expression level of the Ezrin gene with clinical stage and metastasis of osteosarcoma were analyzed using SPSS 16.0 software.

Results: The CTC counts correlated significantly with Enneking stage $(P<0.001)$. The ratio of mesenchymal CTCs correlated with the distant metastases $(P<0.001)$. Ezrin gene expression in CTCs correlated significantly with distant metastases $\left(\chi^{2}=152.51, P=0.000\right)$.

Conclusion: The ratio of mesenchymal CTCs in the peripheral blood of osteosarcoma correlates with distant metastases. High expression of Ezrin gene in CTCs correlates with distant metastases.

Keywords: circulating tumor cells, Ezrin gene, osteosarcoma, metastases

\section{Introduction}

Osteosarcoma is a malignant tumor that threatens the life and health of adolescents; ${ }^{1,2}$ with the introduction of the neoadjuvant chemotherapy, the 5-year survival rate of osteosarcoma patients has increased from $20 \%$ to $60 \%-70 \%{ }^{3,4}$ However, the metastasis of tumor cells and recurrence are still the major reasons for patients' death due to osteosarcoma. ${ }^{5}$ Thus, metastasis and recurrence are difficult problems for osteosarcoma treatment.

A major cause of tumor recurrence could be ascribed to the increasing number of circulating tumor cells (CTCs), especially some chemotherapy-resistant CTCs that may become the origin of relapse after treatment. ${ }^{6}$ Tumor self-seeding by CTCs is also considered to be the reason for tumor metastasis. ${ }^{7}$ Tumor self-seeding involves the CTCs detaching themselves from the primary tumor and entering into the bloodstream, followed by seeding distant organs to form metastases. ${ }^{8}$ Therefore, CTCs could be used to evaluate the prognosis and monitor the therapeutic efficacy of anticancer drugs.

At present, the developed methods for the detection of CTCs can be classified into two major categories: 1) immunochemistry-based methods, including positive selection (enrichment of epithelial marker-positive cells) and negative selection (depletion of CD45-positive blood cells), and 2) physical property-based methods (selection by cell size or electrical charge). ${ }^{9,10}$ Because CTCs from osteosarcoma tumors are mesenchymal in origin and do not express epithelial-specific markers, selection by cell size is used to detect the CTCs from osteosarcoma tumors.
Correspondence: Xia Zhu Department of Orthopaedics, The First Affiliated Hospital of Fujian Medical University, No. 20 Chazhong Road, Fuzhou 350005, Fujian, People's Republic of China

Tel/fax +86 59I 87981029

Email zhuhouy5399@sina.com (c) 1 (1) 2017 Zhong et al. This work is published and licensed by Dove Medical Press Limited. The full terms of this license are available at https://www.dovepress.com/terms.php hereby accept the Terms. Non-commercial uses of the work are permitted without any further permission from Dove Medical Press Limited, provided the work is properly attributed. For permission for commercial use of this work, please see paragraphs 4.2 and 5 of our Terms (https://www.dovepress.com/terms.php). 
Ezrin, an ezrin/radixin/moesin (ERM) family member, is a membrane cytoskeletal linker protein that is known to play an important role in the metastasis of various human cancers, including breast cancer, colorectal carcinoma, gastric cancer and serous ovarian carcinoma. ${ }^{11-15}$ By organizing membrane cytoskeleton-associated complexes and creating specialized membrane domains, Ezrin regulates cellular activities such as survival, adhesion and migration/invasion, all of which are important during tumor development and progression. ${ }^{16-18}$ Furthermore, Ezrin has been suggested to be an important molecule involved in the metastasis of human osteosarcoma. Bulut et a ${ }^{19}$ reported that with the inhibition of Ezrin function by two small molecule inhibitors, the lung metastatic ability of osteosarcoma cells was greatly decreased. Studies by Kim et $\mathrm{al}^{20}$ had shown that pulmonary metastasis occurred in $66.7 \%$ of Ezrin-positive patients, accounting for $90 \%$ of all metastatic patients. Therefore, the expression of Ezrin played an important role in the lung metastasis of osteosarcoma cells.

In this study, we enumerated and characterized the phenotypes of CTCs in the peripheral blood of osteosarcoma patients by using CanPatrol ${ }^{\mathrm{TM}} \mathrm{CTC}$ enrichment technique and detected the expression of Ezrin gene in CTCs by RNA probe technology. Then, the correlations of CTC number, cell type and the expression level of Ezrin gene with clinical stages and metastases were investigated. The results showed that the ratio of mesenchymal CTCs in the peripheral blood of osteosarcoma correlates with distant metastases, and high expression of Ezrin gene in CTCs correlates with distant metastases.

\section{Patients and methods Patients and blood samples}

A total of 41 diagnosed osteosarcoma patients were recruited by The First Affiliated Hospital of Fujian Medical University during January 2015 to March 2016. Written informed consent were obtained from all cancer patients in this study, or patients under 18 years old the consent was obtained from their parents. The classification of cancer stage was according to the Enneking stage. Clinicopathological data were collected, including age, gender, tumor location and tumor clinical stage. All patients' characteristics are summarized in Table 1. The blood samples from our selected patients were collected before surgery. All samples were analyzed with CanPatrol System (SurExam Biotech, Guangzhou, China). ${ }^{21}$

\section{Isolation of CTCs by size}

For the osteosarcoma patients, $5 \mathrm{~mL}$ peripheral blood samples were collected in ethylenediaminetetraacetic acid (EDTA) tubes by venipuncture, and a filtration method was applied using an
Table I Characteristics of patients with osteosarcoma, n (\%)

\begin{tabular}{|c|c|c|c|}
\hline \multirow[t]{2}{*}{ Variables } & \multirow[t]{2}{*}{$\mathbf{n}$} & \multicolumn{2}{|l|}{ CanPatrol $^{\mathrm{TM}}$} \\
\hline & & Positive, n/total (\%) & $P$-value \\
\hline \multicolumn{4}{|l|}{ Age (years) } \\
\hline$<18$ & 26 & $25 / 26(96.1)$ & $0.26 \mathrm{I}$ \\
\hline$\geq 18$ & 15 & 13/15 (86.6) & \\
\hline \multicolumn{4}{|l|}{ Gender } \\
\hline Male & 24 & 22/24 (91.7) & 0.767 \\
\hline Female & 17 & $16 / 17(94.1)$ & \\
\hline \multicolumn{4}{|l|}{ Enneking stage } \\
\hline IA & I & 0 & $<0.001$ \\
\hline $\mathrm{IB}$ & 2 & 0 & \\
\hline IIA & 1 & I & \\
\hline IIB & 26 & 26 & \\
\hline IIIA & 0 & 0 & \\
\hline IIIB & 11 & 11 & \\
\hline \multicolumn{4}{|l|}{ Tumor location } \\
\hline Tibia or femur & 33 & $31 / 33(93.9)$ & 0.53 \\
\hline Other & 8 & $7 / 8(87.5)$ & \\
\hline \multicolumn{4}{|l|}{ Tumor size } \\
\hline$<5 \mathrm{~cm}$ & 11 & 10 & 0.602 \\
\hline$>5 \mathrm{~cm}$ & 30 & 28 & \\
\hline
\end{tabular}

$8 \mu \mathrm{m}$ diameter pores calibrated membrane (Millipore, Billerica, MA, USA). ${ }^{21}$ The required filtration system consisted of a filtration tube containing the membrane (SurExam Biotech), a manifold vacuum plate with valve settings (SurExam Biotech), an E-Z 96 vacuum manifold (Omega, Norcross, GA, USA) and a vacuum pump (Auto Science, Tianjin, China). The red blood cell lysis buffer $\left(154 \mathrm{mM} \mathrm{NH}_{4} \mathrm{Cl}, 10 \mathrm{mM} \mathrm{KHCO}_{3}\right.$ and $0.1 \mathrm{mM}$ EDTA [all from Sigma, St Louis, MO, USA] in deionized water) was used to remove the erythrocytes, then phosphatebuffered saline (PBS) containing 4\% formaldehyde (Sigma) was used to resuspend the remaining cells for 5 minutes before filtration. After transferring the cell suspension to the filtration tube, the pump valve was switched on to reach at least $0.08 \mathrm{MPa}$, and then the filtration began by switching the manifold vacuum plate valve.

\section{Multiplex RNA-in situ hybridization (RNA-ISH) assay}

Three groups of nucleic acid probes were established to identify and examine the expression levels of epithelial and mesenchymal genes in CTCs by a multiplex RNA-ISH assay. Group 1 probes contained four capture probes specific for the epithelial biomarkers EpCAM and cytokeratins (CKs) 8/18/19, and group 2 probes had two capture probes specific for mesenchymal biomarkers vimentin and twist. The last group only contained the capture probe specific for the leukocyte biomarker CD45. The detailed hybridization assay procedure has been presented in the published literature. ${ }^{21}$ Briefly, the 
cells retained on the filter membrane were treated with a protease (Qiagen, Hilden, Germany) before hybridization. Then, the cells were subjected to a series of hybridization reactions with different capture probes, mentioned earlier. Finally, the cells were stained with 4,6-diamidino-2-phenylindole (DAPI). The samples were analyzed with a fluorescence microscope using a 100× oil objective (Olympus BX53; Olympus, Tokyo, Japan). The red and green dots of fluorescent signal observed in the cells represented the epithelial and mesenchymal gene expression, respectively, while the purple fluorescent dots represented the CD45 gene expression (the markers of white blood cells). All sequences were synthesized by Thermo Fisher Scientific (Waltham, MA, USA).

\section{Detection of Ezrin messenger RNA (mRNA) expression level in CTCs}

Ezrin mRNA expression level in CTCs was also detected by RNA-ISH assay. The capture probe specific for Ezrin mRNA was used to capture Ezrin mRNA, followed by conjugation to the branched DNA (bDNA) signal amplification probes to create a branched structure. Finally, the labeled probes conjugated with a fluorescent dye were hybridized to the bDNA sequence. The results were analyzed using a fluorescence microscope.

\section{Statistical analysis}

The data were analyzed using SPSS 16.0 software package (SPSS Inc., Chicago, IL, USA). The correlations of CTC counts with clinical stage were analyzed with $\mathrm{k}$-independentsamples nonparametric tests. The independent-samples $t$-test was used to analyze the correlations of the ratio of mesenchymal CTCs with distant metastases. The correlations of Ezrin expression with distant metastases were analyzed using chi-square test. All data are presented as the mean \pm standard deviation (SD), and $P<0.05$ was considered significant.

\section{Ethics approval}

This study conformed to the ethical guidelines of the Declaration of Helsinki and was approved by The Ethics Committee for Human Research, The First Affiliated Hospital of Fujian Medical University.

\section{Results}

\section{Patient characteristics}

A total of 41 osteosarcoma patients (median age 15 years, range 9-35 years) were enrolled in this study. Of these patients, according to Enneking staging system, eleven patients $(26.8 \%)$ had early metastases as IIIB, 26 patients
(63.4\%) were classified as IIB, one patient (2.4\%) was classified as IIA, two patients ( $5 \%$ ) were classified as IB and one patient $(2.4 \%)$ was classified as IA. CTCs were detected in 41 patients, and the results demonstrated that the positive rate was $92.6 \%(38 / 41)$ (Table 1), while the positive rate was $20 \%$ $(1 / 5)$ in healthy donors (data not shown). Then, the associations of the positive rate of CTCs with clinicopathological variables of osteosarcoma, including age, gender, Enneking stage and tumor location, were analyzed. The results (shown in Table 1) indicated that there was no significant correlation between the positive rate of CTCs and age, gender and tumor location. Significant correlations between the positive rate of CTCs and Enneking stage $(P<0.001)$ were found.

\section{Classification of CTCs}

The CTCs were classified into three subpopulations according to the epithelial-to-mesenchymal transition (EMT) markers by using multiplex RNA-ISH assay, including epithelial CTCs, mesenchymal CTCs and biophenotypic epithelial/mesenchymal CTCs (Figure 1). The results showed that no mesenchymal CTCs and biophenotypic epithelial/ mesenchymal CTCs could be detected in healthy donors (data not shown). In the metastatic stages of osteosarcoma (IIIB), a greater proportion of samples containing mesenchymal CTCs were observed (Table 2). The results showed that the ratio of mesenchymal CTCs in each positive sample increased in the later stages of osteosarcoma compared with the earlier stages of osteosarcoma.

\section{The expression of Ezrin gene in CTCs}

RNA-ISH was applied to investigate the expression of Ezrin in CTCs from 38 osteosarcoma patients. The results showed that Ezrin gene was expressed in $\sim 77.85 \%$ of CTCs. Further research indicated that the expression rates of Ezrin in different types of CTCs were different: $43.9 \%$ in the epithelial CTCs, $68.9 \%$ in the biophenotypic epithelial/mesenchymal CTCs and $81 \%$ in the mesenchymal CTCs; the difference in expression between the epithelial and mesenchymal CTCs was significant (Table S1). The expression levels of Ezrin in stage IIB and stage IIIB were also analyzed; the results showed that the expression levels of Ezrin were different in these two stages. The expression level in stage IIIB was higher than that in stage IIB, indicating that the expression level of Ezrin correlated with the distant metastases (Table 3).

\section{Sensitivity of the CanPatrol system}

The sensitivity and linearity of the CanPatrol system were investigated by spiking 10, 50, 100, 150 and 200 143B cells 

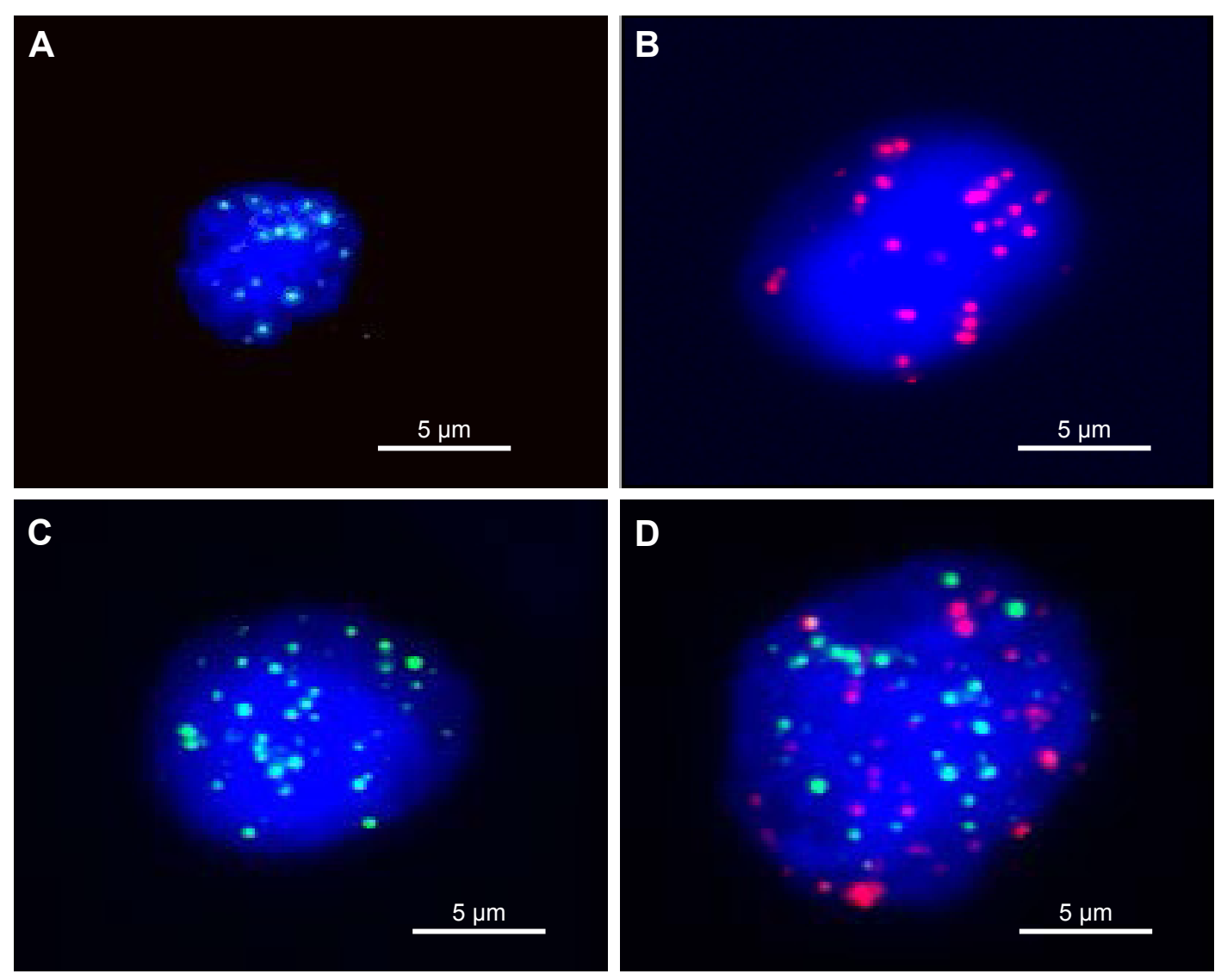

Figure I Fluorescence microscopy images of three types of CTCs isolated from the peripheral blood osteosarcoma patients, based on RNA-ISH staining of epithelial (red dots) and mesenchymal (green dots) markers.

Notes: (A) White blood cells, (B) epithelial CTCs, (C) mesenchymal CTCs, (D) epithelial/mesenchymal CTCs. Scale bar, $5 \mu \mathrm{m}$.

Abbreviations: CTCs, circulating tumor cells; ISH, in situ hybridization.

into $5 \mathrm{~mL}$ of blood to assess recovery of the cells (Figure S1). The correlation coefficient $\left(R^{2}\right)$ was estimated to be 0.999 .

\section{Discussion}

CTCs are cells that have shed into the vasculature from a primary tumor and circulate in the bloodstream..$^{22}$ Due to the noninvasive feature of obtaining sequential blood samples from cancer patients as well as potential clinical application in cancer detection, diagnosis, prognosis and test of drug response, research on CTCs has attracted more and more attention in recent years. ${ }^{23}$ Different techniques have been developed for CTC isolation and characterization, which are based on the cell surface antigens or physical properties of CTCs. ${ }^{24}$ Approaches based on cell surface antigens such as epithelial markers are the most widely applied strategies for CTC detection. Among all the platforms, CellSearch from Janssen Diagnostics is considered the most successful. The practicability of CellSearch has been widely verified by many studies and referred as the "gold standard" for evaluating newly developed approaches. ${ }^{25}$ However, these epithelial antigen-based approaches most likely overlooked the more aggressive CTC subpopulation undergoing EMT. ${ }^{26}$ EMT is

Table 2 Comparison of the CTC counts and the proportion of different CTC types between stage IIB and stage IIIB of patients with osteosarcoma

\begin{tabular}{|c|c|c|c|c|c|}
\hline & IA, IB (n=3) & IIA & IIB $(n=26)$ & IIIB (n=II) & $P$-value \\
\hline CTC counts & 0 & 8 & $24 \pm 6.74$ & $80.72 \pm 14.78$ & 0.00 \\
\hline Ratio of epithelial CTCs (\%) & 0 & 0 & $11.2 \pm 3.97$ & $6.83 \pm 1.37$ & 0.00 \\
\hline Ratio of mesenchymal CTCs (\%) & 0 & 0 & $31.92 \pm 5.35$ & $70.00 \pm 8.27$ & 0.00 \\
\hline Ratio of biophenotypic epithelial/mesenchymal CTCs (\%) & 0 & 0 & $56.87 \pm 6.83$ & $23.15 \pm 4.43$ & 0.00 \\
\hline
\end{tabular}

Note: Data presented as mean \pm standard deviation by calculating the CTC counts per patient in group IIB and IIIB using SPSS software I6. Abbreviation: CTCs, circulating tumor cells. 
Table 3 Different expression levels of Ezrin gene in CTCs of stage IIB and stage IIIB of patients with osteosarcoma

\begin{tabular}{llllll}
\hline $\begin{array}{l}\text { Enneking } \\
\text { stage }\end{array}$ & $\begin{array}{l}\text { CTC } \\
\text { counts }\end{array}$ & $\begin{array}{l}\text { High expression } \\
\text { of Ezrin gene }\end{array}$ & $\begin{array}{l}\text { Medium expression } \\
\text { of Ezrin gene }\end{array}$ & $\begin{array}{l}\text { Low expression } \\
\text { of Ezrin gene }\end{array}$ & $\begin{array}{l}\text { No expression } \\
\text { of Ezrin gene }\end{array}$ \\
\hline IIB & 624 & 124 & 146 & 151 & 203 \\
IIIB & 888 & 423 & 212 & 121 & 132 \\
\hline
\end{tabular}

Abbreviation: CTCs, circulating tumor cells.

a multistep process that plays an important role in metastasis and cancer progression, and CTCs with an EMT phenotype were presumed to be involved in tumor dissemination and metastasis. ${ }^{27,28}$ Considering this phenomenon, the depletion of blood cells may be a supplementary method.

Ezrin belongs to the ERM protein family that acts as membrane organizers and linkers between plasma membrane and cytoskeleton. ${ }^{29}$ Some research has suggested that a high expression of Ezrin was found to be necessary for metastasis in a mouse model of osteosarcoma and a high expression of ezrin in dog osteosarcoma was also associated with early pulmonary metastasis. ${ }^{30-32}$ Thus, it is significant to investigate the relationship between the expression of Ezrin in CTCs and the distant metastases of osteosarcoma.

In this study, the CanPatrol CTC enrichment technique was used to isolate and characterize CTCs of osteosarcoma patients, which could not express epithelial antigens. This technique consists of two major steps: a filtration-based approach (a physical method, an $8 \mu \mathrm{m}$ filtration tube) to isolate CTCs and subsequent characterization of the CTCs using EMT markers, including the epithelial markers EpCAM and CKs and the mesenchymal markers vimentin and twist. ${ }^{21,33}$ EpCAM is a transmembrane glycoprotein that mediates cell-cell adhesion in epithelial tissues, and this protein has oncogenic potential via its capacity to upregulate c-myc, cyclin A and cyclin E. ${ }^{34}$ CKs are the proteins of keratincontaining intermediate filaments found in the cytoskeleton of epithelial cells..$^{21}$ While vimentin is ubiquitously expressed in mesenchymal cells, the expression of vimentin in cancer cells could increase tumor growth and invasiveness. ${ }^{35}$ Twist is a helix-loop-helix protein that is transcriptionally active during cell differentiation. ${ }^{36}$ Our results showed that CTCs were detected in 38 of 41 osteosarcoma patients; the positive rate was $92.6 \%$, reflecting the characteristic of osteosarcoma that was prone to distant metastasis. The results also showed that the ratio of mesenchymal CTCs in each positive sample increased in the later stages of cancer compared with the earlier stages of cancer, and the difference was statistically significant, indicating that mesenchymal CTCs may play an important role in the metastasis of osteosarcoma. This phenomenon could be explained by the theory that the vimentin and twist expressed in the mesenchymal CTCs could upregulate the expression of $\mathrm{N}$-cadherin and downregulate the expression of $\mathrm{N}$-cadherin, which makes the mesenchymal CTCs prone to metastasis. ${ }^{35-37}$ In addition, the results further indicated that the expression levels of Ezrin were different in stage IIB and stage IIIB. The expression level of Ezrin in stage IIIB was higher than that of stage IIB, showing that the expression level of Ezrin correlated with the distant metastases (Table 3).

Due to the relatively small sample size in this study, the association of CTC counts with Enneking stage may be bias. However, we assessed that the ratio of mesenchymal CTCs in each positive sample increased in the later stages of osteosarcoma compared with the earlier stages of osteosarcoma, and the expression level of Ezrin in stage IIIB was higher than that of stage IIB, indicating that the ratio of mesenchymal CTCs and the expression level of Ezrin in CTCs were associated with distant metastases of osteosarcoma.

\section{Conclusion}

This study provided a novel approach for isolation and characterization of CTCs from osteosarcoma patients by a combination of physical and biological methods. Our findings have provided evidence of the EMT-like phenomenon in CTCs of osteosarcoma, and the ratio of mesenchymal CTCs was associated with distant metastases of osteosarcoma. Furthermore, we demonstrated that the expression level of Ezrin in CTCs was also associated with distant metastases of osteosarcoma. Further studies could be performed to investigate the correlation of the ratio of mesenchymal CTCs with progression-free survival and overall survival of osteosarcoma.

\section{Acknowledgments}

The authors gratefully acknowledge the financial support of the National Natural Science Foundation of China (81301520), the Natural Science Foundation of Fujian Province (2016J01540, 2014J05088), the outstanding youth scientific research personnel training plan of colleges and universities in Fujian Province (2015B027), Joint Funds for the Innovation of Science and Technology, Fujian Province (2016Y9019), the outstanding youth fund of Fujian 
Province, the special fund of youth top-notch innovative talents of Fujian Province (SQNBJ201601) and the Key Clinical Specialty Discipline Construction Program of Fujian Province, People's Republic of China (2012-SLCZD-1).

\section{Disclosure}

The authors report no conflicts of interest in this work.

\section{References}

1. Luetke A, Meyers PA, Lewis I, Juergens H. Osteosarcoma treatment - where do we stand? A state of the art review. Cancer Treat Rev. 2014;40(4):523-532.

2. Leary SES, Wozniak AW, Billups CA, et al. Survival of pediatric patients after relapsed osteosarcoma: The St Jude Children's Research Hospital experience. Cancer. 2013;119(14):2645-2653.

3. Ogura K, Fujiwara T, Yasunaga H, et al. Development and external validation of nomograms predicting distant metastases and overall survival after neoadjuvant chemotherapy and surgery for patients with nonmetastatic osteosarcoma: a multi-institutional study. Cancer. 2015;121(21): 3844-3852.

4. Collins M, Wilhelm M, Conyers R, et al. Benefits and adverse events in younger versus older patients receiving neoadjuvant chemotherapy for osteosarcoma: findings from a meta-analysis. J Clin Oncol. 2013; 31(18):2303-2312.

5. Briccoli A, Rocca M, Salone M, Guzzardella GA, Balladelli A, Bacci G. High grade osteosarcoma of the extremities metastatic to the lung: long-term results in 323 patients treated combining surgery and chemotherapy, 1985-2005. Surg Oncol. 2010;19(4):193-199.

6. Mitra A, Mishra L, Li S. EMT, CTCs and CSCs in tumor relapse and drug-resistance. Oncotarget. 2015;6(13):10697-10711.

7. Rice J. Metastasis: the rude awakening. Nature. 2012;485:S55-S57.

8. Kim MY, Oskarsson T, Acharyya S, et al. Tumor self-seeding by circulating cancer cells. Cell. 2009;139:1315-1326.

9. Joosse SA, Pantel K. Biologic challenges in the detection of circulating tumor cells. Cancer Res. 2013;73(1):8-11.

10. Alix-Panabières C, Pantel K. Challenges in circulating tumour cell research. Nat Rev Cancer. 2014;14:623-631.

11. Fiévet B, Louvard D, Arpin M. ERM proteins in epithelial cell organization and functions. Biochim Biophys Acta. 2007;1773(5):653-660.

12. Hoskin V, Szeto A, Ghaffari A, Greer PA, Côté GP, Elliott BE. Ezrin regulates focal adhesion and invadopodia dynamics by altering calpain activity to promote breast cancer cell invasion. Mol Cell Biol. 2015; 26(19):3464-3479.

13. Leiphrakpam PD, Rajput A, Mathiesen M, et al. Ezrin expression and cell survival regulation in colorectal cancer. Cell Signal. 2014; 26(5):868-879.

14. Li L, Wang YY, Zhao ZS, Ma J. Ezrin is associated with gastric cancer progression and prognosis. Pathol Oncol Res. 2011;17(4):909-915.

15. Köbel M, Gradhand E, Zeng K, et al. Ezrin promotes ovarian carcinoma cell invasion and its retained expression predicts poor prognosis in ovarian carcinoma. Int J Gynecol Pathol. 2006;25(2):121-130.

16. Bretscher A, Edwards K, Fehon RG. ERM proteins and merlin: integrators at the cell cortex. Nat Rev Mol Cell Biol. 2002;3(8):586-599.

17. Srivastava J, Elliott BE, Louvard D, Arpin M. Src-dependent Ezrin phosphorylation in adhesion-mediated signaling. Mol Biol Cell. 2005; 16(3):1481-1490.
18. Sarrió D, Rodríguez-Pinilla SM, Dotor A, Calero F, Hardisson D, Palacios J. Abnormal ezrin localization is associated with clinicopathological features in invasive breast carcinomas. Breast Cancer Res Treat. 2006;98(1):71-79.

19. Bulut G, Hong SH, Chen K, et al. Small molecule inhibitors of ezrin inhibit the invasive phenotype of osteosarcoma cells. Oncogene. 2012; 31(3):269-281.

20. Kim C, Shin E, Hong S, et al. Clinical value of Ezrin expression in primary osteosarcoma. Cancer Res Treat. 2009;41(3):138-144.

21. Wu S, Liu S, Liu Z, et al. Classification of circulating tumor cells by epithelial-mesenchymal transition markers. PLoS One. 2015; 10(4):e0123976.

22. Gupta GP, Massagué J. Cancer metastasis: building a framework. Cell. 2006;127(4):679-695.

23. Pavese JM, Bergan RC. Circulating tumor cells exhibit a biologically aggressive cancer phenotype accompanied by selective resistance to chemotherapy. Cancer Lett. 2014;352(2):179-186.

24. Chen XX, Bai F. Single-cell analyses of circulating tumor cells. Cancer Biol Med. 2015;12(3):184-192.

25. Riethdorf S, Fritsche H, Muller V, et al. Detection of circulating tumor cells in peripheral blood of patients with metastatic breast cancer: a validation study of the CellSearch system. Clin Cancer Res. 2007;13(3):920-928.

26. Konigsberg R, Obermayr E, Bises G, et al. Detection of EpCAM positive and negative circulating tumor cells in metastatic breast cancer patients. Acta Oncol. 2011;50(5):700-710.

27. Thiery JP, Acloque H, Huang RY, Nieto MA. Epithelial-mesenchymal transitions in development and disease. Cell. 2009;139:871-890.

28. Kang Y, Pantel K. Tumor cell dissemination: emerging biological insights from animal models and cancer patients. Cancer Cell. 2013; 23(5):573-581.

29. Mangeat $P$, Roy C, Martin M. ERM proteins in cell adhesion and membrane dynamics. Trends Cell Biol. 1999;9(5):187-192.

30. Khanna C, Wan XL, Bose SL, et al. The membrane cytoskeleton linker ezrin is necessary for osteosarcoma metastasis. Nat Med. 2004; 10:182-186.

31. Wang YF, Shen JN, Xie XB, Wang J, Huang G. Expression change of ezrin as a prognostic factor in primary osteosarcoma. Med Oncol. 2011;28(suppl 1):S636-S643.

32. Salas S, Bartoli C, Deville JL, et al. Ezrin and alpha-smooth muscle actin are immunohistochemical prognostic markers in conventional osteosarcomas. Virchows Arch. 2007;451(6):999-1007.

33. Li YM, Xu SC, Li J, et al. Epithelial-mesenchymal transition markers expressed in circulating tumor cells in hepatocellular carcinoma patients with different stages of disease. Cell Death Dis. 2013;4:e831.

34. Osta WA, Chen Y, Mikhitarian K, et al. EpCAM is overexpressed in breast cancer and is a potential target for breast cancer gene therapy. Cancer Res. 2004;64(16):5818-5824.

35. Zhao Y, Yan Q, Long X, Chen X, Wang Y. Vimentin affects the mobility and invasiveness of prostate cancer cells. Cell Biochem Funct. 2008; 26(5):571-577.

36. Yang J, Mani SA, Donaher JL, et al. Twist, a master regulator of morphogenesis, plays an essential role in tumor. Metastasis Cell. 2004;117(7):927-939.

37. Yang GQ, Yuan J, Li KH. EMT transcription factors: implication in osteosarcoma. Med Oncol. 2013;30(4):697. 


\section{Supplementary materials}

Table SI The expression rates of the Ezrin gene in different types of CTCs

\begin{tabular}{lll}
\hline Types of CTCs & $\begin{array}{l}\text { Positive for } \\
\text { Ezrin gene (\%) }\end{array}$ & $\begin{array}{l}\text { Negative for } \\
\text { Ezrin gene (\%) }\end{array}$ \\
\hline Epithelial CTCs & 43.9 & 56.1 \\
Mesenchymal CTCs & 68.9 & 31.1 \\
Biophenotypic epithelial/ & 81 & 19 \\
mesenchymal CTCs & & \\
\hline
\end{tabular}

Abbreviation: CTCs, circulating tumor cells.

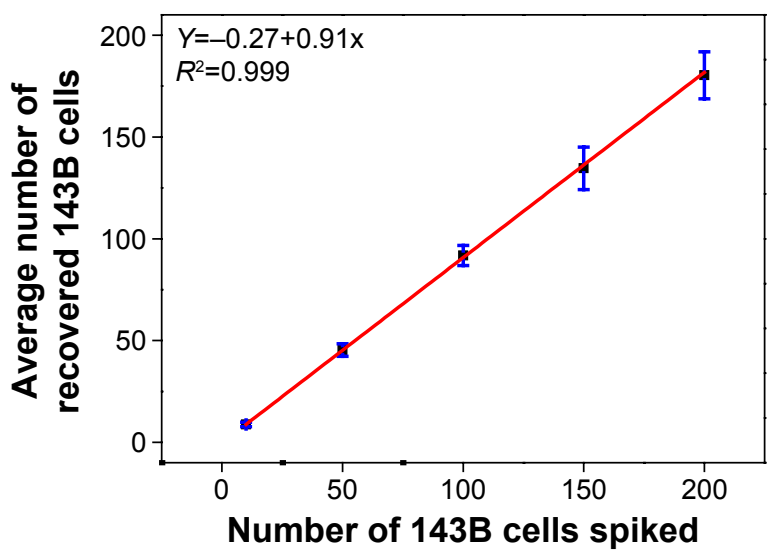

Figure SI Calibration curve obtained using the CanPatrol ${ }^{\mathrm{TM}}$ СTC enrichment technique in the spiking experiment $(n=5)$ using 143B cells at different dilutions. Abbreviation: CTCs, circulating tumor cells.

\section{Publish your work in this journal}

OncoTargets and Therapy is an international, peer-reviewed, open access journal focusing on the pathological basis of all cancers, potential targets for therapy and treatment protocols employed to improve the management of cancer patients. The journal also focuses on the impact of management programs and new therapeutic agents and protocols on
Dovepress

patient perspectives such as quality of life, adherence and satisfaction. The manuscript management system is completely online and includes a very quick and fair peer-review system, which is all easy to use. Visit http://www.dovepress.com/testimonials.php to read real quotes from published authors. 